\title{
ASPECTOS EPIDEMIOLÓGICOS E O CUIDADO DE ENFERMAGEM NA TENTATIVA DE SUICÍDIO
}

\author{
EPIDEMIOLOGICAL ASPECTS AND \\ NURSING CARE IN SUICIDE ATTEMPTS
}

\begin{abstract}
Eliany Nazaré Oliveira*, Tamires Alexandre Felix**, Camila Bezerra de Lima Mendonça***, Paulo Sérgio Ferreira Lima***, Adeliane Souza Freire****, Roberta Magda Martins Moreira****
\end{abstract}

Autor para correspondência: Camila Bezerra de Lima Mendonça - jully_mila@hotmail.com

*Enfermeira. Doutora em enfermagem pela Universidade Federal do Ceará - UFC. Docente do curso de graduação em enfermagem da Universidade Estadual Vale do Acaraú - UVA. Sobral-CE-Brasil.

**Enfermeira. Mestra em Saúde da Família. Especialista em Gestão dos Serviços de Urgência e Emergência. Especialista em Terapia Intensiva.

***Graduado(a) em Enfermagem.

****Discente do curso de enfermagem pela Universidade Estadual Vale do Acaraú (UVA). Pesquisadora bolsista da FUNCAP. Sobral-CE-Brasil.

\section{R E S U M O}

Objetivou-se traçar o perfil epidemiológico descritivo de pacientes atendidos numa unidade de emergência de um hospital de referência na zona norte do estado do Ceará/Brasil com o diagnóstico de tentativa de suicídio e conhecer o cuidado de enfermagem prestado a estes. Trata-se de um estudo transversal, descritivo-exploratório. Foram abordados 60 casos através de um formulário próprio. Os dados foram processados e analisados através programa Epi-Info. Atendeu-se a Resolução 466/12 do Conselho Nacional de Saúde. A cidade de Sobral teve o maior índice de admissões. A maioria dos casos ocorreu com mulheres jovens, solteiras que utilizaram a intoxicação exógena como método de autoextermínio. Quanto à ocupação, destacaram-se desempregados, estudantes e agricultores. Observou-se o uso de álcool e outras drogas associados. $40 \%$ dos pacientes eram reincidentes. Entre as motivações mais relatadas estão os conflitos familiares e amorosos. A metade dos pacientes classificou - cuidado de enfermagem como Bom. Em geral, a maioria das intervenções realizadas nos pacientes internados estava ligadas a procedimentos técnicos de enfermagem. Pesquisas paralelas de caráter qualitativo podem ser desenvolvidas para esclarecer melhor este agravo.

PALAVRAS-CHAVE: Tentativa de Suicídio; Epidemiologia; Enfermagem. 
This study aimed to trace the descriptive epidemiological profile of patients cared for in the emergency department of a referral hospital following suicide attempts. This is a cross study, descriptive-exploratory. Sixty $(60)$ cases were assessed. The data was processed and analyzed with Epi-Info. The services were in compliance with the Resolution 466/12 of the Brazilian National Health Council. Sobral city had the highest admissions rate. Most cases occurred among single unemployed - students and farmers - young women, by exogenous intoxication. It was observed the use of alcohol and other drugs. $40 \%$ of them tried to commit suicide more than once. Among the most frequently reported motivations were relationships conflicts - romantic and family wise. Half of the patients classified the nursing care as good. Qualitative research can be developed parallel to clarify this situation.

KEYWORDS: Suicide Attempts; Epidemiology; Nursing. 
Para a Organização Mundial de Saúde (OMS), suicídio constitui-se em um ato deliberado iniciado e levado a cabo por uma pessoa com pleno conhecimento ou expectativa de um resultado fatall. O suicídio como violência auto-infligida está, na maioria dos países, entre as dez principais causas de morte e entre as mais frequentes em adolescentes e adultos jovens, constituindo-se em um grave problema de saúde pública.

O Brasil encontra-se no grupo de países com baixa taxa de suicídio, mas como é um país populoso atinge o nono lugar em números absolutos. Além do suicídio em si, há outro problema a ele ligado: o elevado número de pessoas que tentam o suicídio. Registros oficiais sobre tentativas de suicídio são escassos, porém estima-se que o número supere $\circ$ número de suicídios em pelo menos dez vezes ${ }^{2}$.

Estudo refere dados da OMS que indicam para o ano de 2020 aproximadamente 1,53 milhão de mortes por suicídio e um número de 10 a 20 vezes maior de tentativas. Isso representa uma morte a cada 20 segundos e uma tentativa de suicídio a cada meio segundo. No Brasil, a taxa oficial de suicídio varia em torno de 4,1 por $100 \mathrm{mil} /$ habitantes $^{3}$.

No Ceará, as taxas de mortalidade relacionadas a agressões autodirigidas registram ascensão significativa ${ }^{4}$. Um estudo ecológico de Oliveira (2010) realizado no Ceará constatou o aumento de 265 ocorrências de suicídio-ano em 1998 para 525 ocorrências de suicídio-ano em 2007 e uma variação na taxa de 3,8 para 6,3/100.000 no mesmo período, o que corresponde a um crescimento de $65,79 \%$ na taxa de suicídio, enquanto o crescimento da população neste mesmo período correspondeu a $18,66 \%$.

Com relação ao cuidado dos pacientes após a tentativa de suicídio, mais especificamente no ambiente de emergência, sabe-se que esta primeira abordagem está diretamente relacionada à ocorrência de novas tentativas e ao seguimento referenciado do paciente na Rede de Atenção Psicossocial visando a diminuir a exposição aos fatores de risco e desenvolver, em âmbito familiar e social, ações de promoção à saúde e valorização da vida.

Para a categoria dos profissionais de enfermagem, que atua na emergência e em todos os demais pontos da Rede, traçar ações específicas para esta demanda tem sido um desafio. Esse fato tem instigado pesquisadores oriundos de vários campos da área científica. Mesmo assim, poucos estudos têm descrito o fenômeno, seja no aspecto médico, epidemiológico ou social. Daí surge à importância de se investigar o assunto por meio de pesquisas que revelem a magnitude do agravo e caracterizem os casos possibilitando intervenções da gestão em saúde e o aperfeiçoamento do cuidado.

O presente estudo tem como objetivo traçar o perfil epidemiológico descritivo de pacientes atendidos numa unidade de emergência em um hospital de referência da mesorregião Noroeste do Ceará com - diagnóstico de Tentativa de Suicídio e identificar - julgamento do paciente/família sobre o cuidado de enfermagem prestado neste setor.

\section{VIOLÊNCIA AUTODIRIGIDA E DIRETRIZES DE CUIDADO}

\begin{abstract}
O suicídio é um fenômeno determinado biopsicologicamente influenciado por aspectos socioculturais que cercam cada indivíduo. Tratase de um percurso que vai desde a motivação e ideação suicida até o planejamento do método e a autoagressão.
\end{abstract}

Diversas circunstâncias podem aumentar o risco de suicídio por serem produtoras de estresse, como: desemprego, pobreza, perda de um ente querido, desentendimentos com familiares e amigos, término de uma relação afetiva, problemas legais ou de trabalho, uso abusivo de álcool e outras drogas, disponibilidade dos meios para efetuar o ato suicida, violência física e/ou sexual na infância, isolamento social e distúrbios psíquicos, como a depressão ou a esquizofrenia ${ }^{5}$. As diferentes formas de lidar com este estresse e com a perda de sentido na vida podem disparar em algumas pessoas pensamentos de morte. 
Mediante esta realidade em 2006, 。 Ministério da Saúde instituiu as Diretrizes Nacionais para Prevenção do Suicídio, que destacam, entre seus objetivos, o desenvolvimento de estratégias de promoção da saúde mental, qualidade de vida, educação, proteção, recuperação da saúde e prevenção de danos. Estas diretrizes incentivam a coleta de dados epidemiológicos sobre os casos de suicídio, bem como a elaboração de projetos que possam qualificar a prestação de serviços a indivíduos que tentam contra a própria vidab .

- Manual de Prevenção ao Suicídio (2006), também se constitui de um normativo ministerial dirigido a profissionais da saúde com o intuito de transmitir informações básicas que possam orientar a detecção precoce do comportamento suicida e - manejo inicial de pessoas que se encontrem sob risco auxiliando no desenvolvimento de ações em diversos níveis de atenção ${ }^{7}$.

Sob este prisma, é possível inferir que as ações estão ainda limitadas para combater este agravo. É imperativo ampliar a produção do conhecimento sobre o impacto do comportamento suicida nas famílias, os aspectos próprios do cuidar de cada uma das profissões de saúde, os estigmas sociais que dificultam a reabilitação dos pacientes e $\circ$ adequado uso dos registros.

\section{MÉTODO}

Trata-se de um estudo Quantitativo, Transversal, Descritivo Exploratório que compõe a pesquisa 'Saúde Mental e Cuidado de Enfermagem à Pessoa que Tentou Suicídio'. A coleta de dados foi realizada na unidade de emergência adulta da Santa Casa de Misericórdia de Sobral (CE) referência para casos de tentativa de suicídio na mesorregião Noroeste do Estado. Os participantes do estudo foram todos aqueles admitidos na unidade no período de julho de 2013 a maio de $2014 \mathrm{com}$ diagnostico de Tentativa de Suicídio de acordo com - CID-10 e que aceitaram participar da pesquisa formalizando o consentimento por meio do Termo de Consentimento Livre e Esclarecido assinado pelo paciente ou responsável. Para classificação dos suicídios foi utilizada a Décima Segunda Revisão da Classificação Internacional de Doenças (CID-10) ${ }^{8}$.

Foram coletados 60 casos e excluídos 23 devido à: óbito (05); alta melhorada em menos de 12 horas (10); transferência para outro setor $(01)$; recusa em participar (03); alta a pedido (02); transferência para outro hospital (01) e evasão (01).

A coleta de dados foi realizada a partir de um formulário próprio preenchido com base nas informações contidas nos prontuários e através de uma entrevista breve semiestruturada com o paciente / familiar responsável.

Os achados da pesquisa foram organizados e processados através do programa Epi-Info versão 3.5.1 sendo posteriormente analisados por métodos bioestatísticos. Procedeu-se conforme a Resolução 466/12 do Conselho Nacional de Saúde (CNS) ${ }^{9}$. Obteve-se aprovação do Comitê de Ética da Universidade Estadual Vale do Acaraú com parecer $n^{\circ} 384.646$.

Tabela 1 - Distribuição dos casos de tentativa de suicídio atendidos na Santa Casa de Misericórdia de Sobral, segundo as características sociodemográficas. Sobral, CE, Brasil, 2015.

\begin{tabular}{|c|c|c|}
\hline Características Sóciodemográficas & $\mathbf{N}$ & $\%$ \\
\hline \multicolumn{3}{|l|}{ Sexo } \\
\hline Masculino & 21 & $35,0 \%$ \\
\hline Feminino & 39 & $65,0 \%$ \\
\hline Total & 60 & $100 \%$ \\
\hline \multicolumn{3}{|l|}{ Faixa Etária } \\
\hline Entre 12 e 21 & 24 & $40,0 \%$ \\
\hline Entre 21 e 41 & 27 & $45,0 \%$ \\
\hline Entre 41 e 61 & 8 & $13,3 \%$ \\
\hline Acima de 61 & 1 & $1,7 \%$ \\
\hline Total & 60 & $100 \%$ \\
\hline \multicolumn{3}{|l|}{ Estado Civil } \\
\hline Solteiro & 25 & $41,7 \%$ \\
\hline União Estável & 20 & $33,3 \%$ \\
\hline Casado & 14 & $23,3 \%$ \\
\hline Viúvo & 1 & $1,7 \%$ \\
\hline Total & 60 & $100 \%$ \\
\hline \multicolumn{3}{|l|}{ Nível de Escolaridade } \\
\hline Analfabeto & 4 & $6,7 \%$ \\
\hline Ensino Fundamental & 30 & $50,0 \%$ \\
\hline Ensino Médio & 20 & $33,3 \%$ \\
\hline Ensino Superior & 3 & $5,0 \%$ \\
\hline Ignorado & 3 & $5,0 \%$ \\
\hline Total & 60 & $100 \%$ \\
\hline \multicolumn{3}{|c|}{ Recebe algum apoio social/governamental } \\
\hline Sim & 21 & $35,0 \%$ \\
\hline Não & 39 & $65,0 \%$ \\
\hline Ignorado & 0 & 0 \\
\hline Total & 60 & $100 \%$ \\
\hline
\end{tabular}

As variáveis: uso abusivo/dependência de crack, álcool e outras drogas; ocorrência de tentativa anterior; histórico de suicídio na família; ser portador 
de alguma desordem mental comportaram-se como fatores de risco elevando potencialmente a possibilidade de autoagressão. Denominar-se de alguma religião ou desenvolver práticas de apoio espiritual, além de atividades de lazer não se mostraram fatores protetores como a literatura apresenta.

Tabela 2 - Distribuição dos Casos de Tentativa de Suicídios atendidos na Santa Casa de Misericórdia de Sobral quanto aos determinantes sociais e clínicos. Sobral, CE, Brasil, 2015.

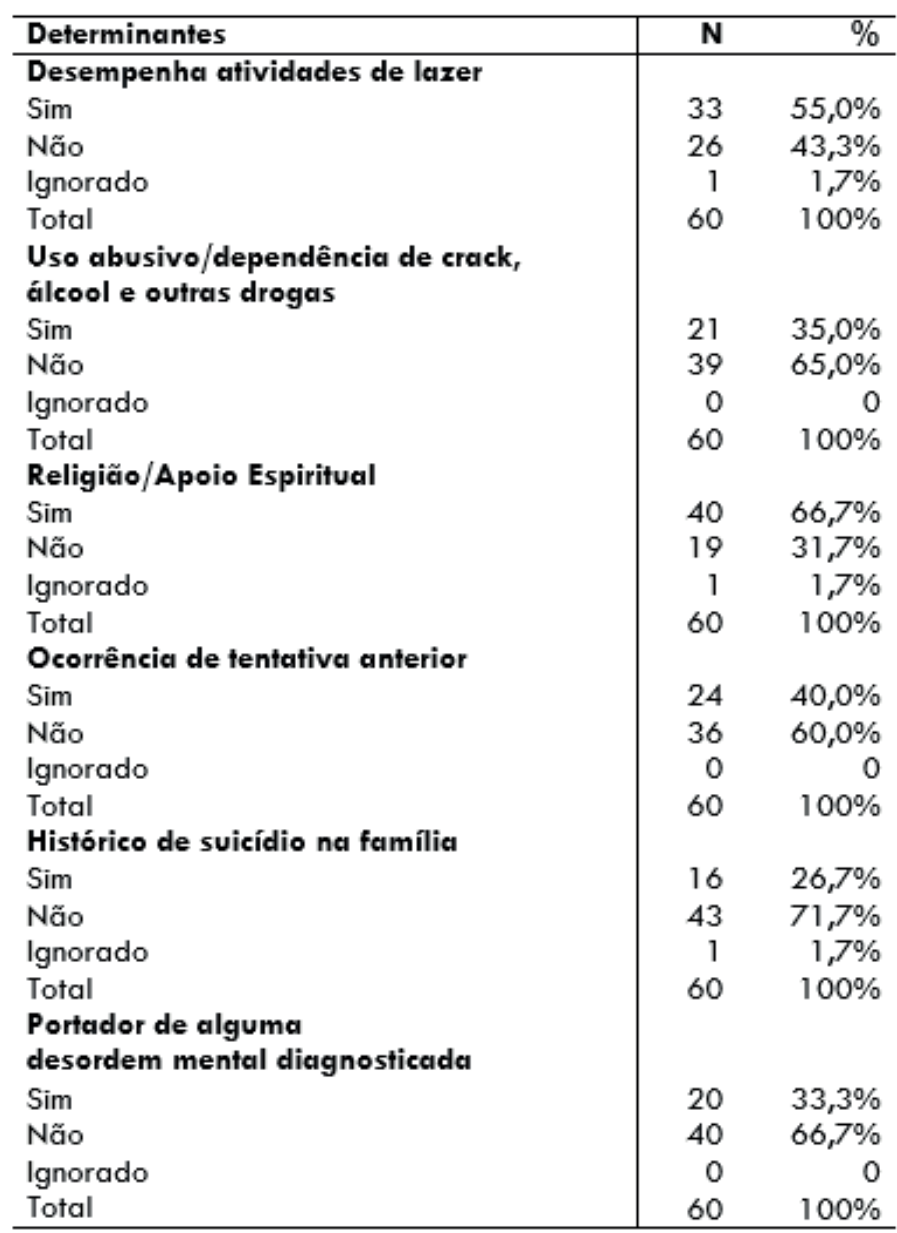

- método utilizado foi predominantemente a Intoxicação Exógena predizendo intervenções urgentes no campo da educação em saúde, comercialização de tóxicos e prevenção na atenção primária.

Tabela 3 - Distribuição dos Casos de Tentativa de Suicídios atendidos na Santa Casa de Misericórdia de Sobral, segundo o diagnóstico. Sobral, CE, Brasil, 2015.

\begin{tabular}{l|rr}
\hline Diagnósticos & $\mathbf{N}$ & $\%$ \\
\hline Intoxicação Exógena & 52 & $86,7 \%$ \\
Enforcamento & 3 & $5,0 \%$ \\
Ferimento por Arma Branca & 1 & $1,7 \%$ \\
Queimaduras & 1 & $1,7 \%$ \\
Outros Tipos & 3 & $5,0 \%$ \\
Total & 60 & $100 \%$ \\
\hline
\end{tabular}

A Tabela 4 apresenta a avaliação do paciente/ familiar sobre os cuidados de enfermagem incluindo apoio psicológico, postura ética, procedimentos técnicos e encaminhamento na Rede de Atenção Psicossocial.

Tabela 4 - Distribuição dos Casos de Tentativa de Suicídios atendidos na Santa Casa de Misericórdia de Sobral, segundo a avaliação do paciente/ familiar sobre os Cuidados de Enfermagem. Sobral, CE, Brasil, 2015.

\begin{tabular}{l|rr}
\hline Cuidado de Enfermagem & N & $\%$ \\
\hline Ruim & 4 & $6,7 \%$ \\
Regular & 7 & $11,7 \%$ \\
Bom & 30 & $50,0 \%$ \\
Ótimo & 17 & $28,3 \%$ \\
Não sabe & 2 & $3,3 \%$ \\
Total & 60 & $100 \%$ \\
\hline
\end{tabular}

\section{MÉTODO}

Os resultados apontam Sobral, cidade sede da macrorregião, como procedência da maioria dos casos de tentativa de suicídio $(38,3 \%)$; dado bastante relevante considerando que a unidade de emergência é referência para esses casos em todo território que compreende cerca de 55 municípios. Esta expressividade pode estar relacionada à maior densidade demográfica do município ou à proximidade dos pacientes com relação ao hospital facilitando os encaminhamentos. Tais considerações também despertam ao fato da comercialização aberta de venenos muito utilizados nas tentativas de suicídio e para um possível déficit no acompanhamento de pacientes com fatores de risco presentes, visto que a cidade possui os serviços substitutivos propostos pela política de saúde mental brasileira, tais como CAPS, ambulatório psiquiátrico e equipes multiprofissionais atuando na Estratégia Saúde da Família.

A prevalência de adolescentes e adultos jovens na amostra também gera uma discussão sobre a falta de informações e preparo emocional, baixa escolaridade e frágil estrutura familiar por risco social comum nesta faixa etária como fatores determinantes na ocorrência de autoagressão nesta região. 
A análise das variáveis conjugadas relacionadas ao perfil sócio-demográfico (Tabela 1) revelou que os casos ocorrem em sua maioria com mulheres jovens, solteiras e sem apoio social. Alguns estudos corroboram este resultado afirmando que geralmente as mulheres tentam mais o suicídio, enquanto que nos homens essa tentativa culmina, na maioria das vezes, em morte. Isto ocorre porque os homens procuram meios mais letais para a tentativa do suicídio como arma de fogo, enforcamento, uso de arma branca, ingestão de substâncias nocivas, enquanto que as mulheres cometem a tentativa por intoxicação exógena e outros meios de menor letalidade ${ }^{10}$.

Quanto à ocupação dos pacientes, a maioria dos casos ocorreu entre desempregados, estudantes e agricultores. Esse dado converge com a falta de apoio social, a faixa etária predominantemente jovem e ao fácil acesso a agrotóxicos respectivamente.

Remete-se, portanto, a falta de apoio social que segundo Alves e Rodrigues ${ }^{11}$ consiste na omissão dos governos em reduzir estressores relacionados ao ambiente social como meios de transporte, saneamento básico, oportunidades de emprego, habitação digna, redução da violência e apoio financeiro para tratamento psiquiátrico.

É consenso na literatura apresentar o lazer e a religião como fatores protetores contra o percurso suicida. Portanto, o que se espera é que estas variáveis se relacionem de forma inversamente proporcional com a agressão autodirigida ${ }^{12}$. De maneira divergente, este estudo retratou que 55\% dos casos de tentativas de suicídio desempenham com frequência atividades de lazer como sair com os amigos, frequentar festas, praticar esportes, ir ao cinema e $66,7 \%$ dos casos afirmaram ter apoio espiritual e desenvolver práticas religiosas rotineiramente. Podem-se associar estes valores ao fato de que a maioria dos casos constitui-se de primeira tentativa geralmente por impulso emocional jovem não caracterizando um ato idealizado e planejado.

Dos 60 casos, 21 referiram uso abusivo/dependência de crack, álcool e outras drogas. Este dado indica a relação direta do uso de drogas na determinação da tentativa de suicídio. Muitos dos pacientes referiram estar embriagados ao cometer, como se costuma chamar, 'o último ato'.

Estudo investigando 84 serviços públicos de emergência distribuídos pelo Brasil, em 2007, por 30 dias, registrou 516 atendimentos por tentativa de suicídio, sendo que $25 \%$ dos pacientes relataram uso abusivo de drogas diversas. Isto se relaciona à desesperança e a situação constrangedora de atrelar a vida ao consumo de substâncias que ocasionam sofrimentos psíquicos intensos no núcleo familiar gerando pensamentos de autodestruição ${ }^{13}$. Considerando os três principais determinantes da tentativa de suicídio: ocorrência de tentativa anterior, histórico de suicídio na família e em pessoas próximas e ser portador de transtornos mentais, mais especificamente a Depressão, a pesquisa apresentou resultados condizentes com estudos semelhantes já publicados ${ }^{14-15}$.

É notável o fato de que $40 \%$ dos pacientes tentaram suicídio mais de uma vez levando a discussão sobre um acompanhamento possivelmente ineficaz nos serviços de saúde mental e na Atenção Primária à Saúde, bem como a prevalência dos estressores relacionados com a autoagressão. Estudos chamam a atenção para o fato de que a probabilidade de tentativas aumenta depois da primeira, elevando, assim, o risco de consumação do suicídio ${ }^{16}$.

Os pacientes que tiveram tentativas ou óbitos por suicídio em pessoas próximas nos últimos cinco anos relataram um ambiente mórbido e a perda de entes queridos como fatores idealizadores do suicídio. A busca por 'encontrar o ente querido que morreu' em outros planos de vida foi referida algumas vezes durante os encontros. 'A morte que induz a morte'.

Para os pacientes portadores de transtornos mentais $(n=20)$ ressalta-se um possível viés relacionado aos pacientes com clinica nitidamente depressiva, porém ainda não diagnosticados e aqueles que referiram transtornos psiquiátricos, sem um diagnóstico médico. Outro fato que merece destaque, percebido nos depoimentos, é a recusa de tratamento. Muitos deles nutrem preconceito ao fato de ser "acompanhado no CAPS", considerando o comportamento anormal como passageiro e os conflitos familiares e amorosos como contexto particular que não pertence aos profissionais de saúde.

O diagnóstico indicado para $86,7 \%$ dos casos é de Intoxicação Exógena (Tabela 3) em sua maioria por 
agrotóxicos, raticidas, produtos de limpeza e por medicações de uso contínuo do próprio paciente, em grande parte dos casos, ansiolíticos. Em seguida, surgem os enforcamentos e os métodos combinados onde $\circ$ paciente aplica mais de um ato violento autodirigido sequenciado.

Estas intoxicações apresentaram-se em sua maioria leves; o tempo de busca por atendimento é curto e o suporte de emergência eficaz o que permite a evolução da maioria dos casos com alta melhorada sem sequelas $(56,7 \%)$. Da amostra houve um paciente que evoluiu para óbito, $20 \%$ foram encaminhados para outros setores para manter tratamento clínico, inclusive terapia intensiva, e outros foram contra referenciados ao hospital de origem ou encaminhados para internação psiquiátrica.

Os sentimentos mais referidos foram insegurança, angústia e fragilidade com baixas perspectivas de futuro e medo de repressões familiares, bem como de discriminação social. Outro fato relevante é a causa dessas tentativas de suicídio; a maioria relatou tentar contra a própria vida motivados por conflitos familiares e amorosos.

Os aspectos subjetivos intrínsecos à tentativa de suicídio são obtidos sob uma lógica conflituosa no que tange ao sentido da vida e a autopercepção. É preciso considerar o contexto em que foram coletados os dados permitindo declarações que podem simular realidades em busca de alta prévia ou apoio dos familiares.

Quanto ao cuidado de enfermagem, mesmo apresentando dados positivos, destaca-se a necessidade de qualificar o atendimento por meio da elaboração de protocolos assistenciais para os pacientes e seus familiares. Afinal, a enfermagem deve estar qualificada a prestar um atendimento ético, eficaz, reestabelecendo fisicamente $\circ$ paciente à medida que fornece apoio psicológico priorizando a escuta qualificada e um cuidado livre de preconceitos ou julgamentos de valor ${ }^{17}$.

Visualizou-se que a maioria das intervenções realizadas nos pacientes internados estavam ligadas a procedimentos técnicos de enfermagem como utilização de sondas gástricas para drenagem de substâncias nocivas, uso de medicação endovenosa por meio de cateter periférico, monitorização contínua e encaminhamento para exames havendo poucas intervenções associadas à saúde mental do indivíduo, abordagem familiar ou cuidados multiprofissionais.

A maioria dos pacientes relatou manter ideação suicida, estar frustrado pela falta de valorização da própria vida. A coleta de dados, ao contrário do que se esperava, não se mostrou dolorosa aos pacientes, mas converteu-se num momento de expressão, escuta e apoio interpessoal.

\section{CONCLUSÃO}

Neste contexto, é fundamental a aplicação de práticas desenvolvidas especificamente para este público, pois a pessoa que tenta suicídio, ao receber $\circ$ primeiro atendimento em uma unidade de saúde, encontra-se em um momento de extrema vulnerabilidade.

Dispara-se à necessidade de investimento em políticas públicas que supram as carências dos serviços de emergência e saúde mental, visando melhores condições de trabalho para os profissionais em questão, assim como para os usuários além de fortalecer as perspectivas de Rede para garantir 0 acompanhamento dos grupos de risco. Nem todos os casos de suicídio podem ser prevenidos, entretanto muitas vidas serão salvas se todas as pessoas que tentaram $\circ$ suicídio forem adequadamente abordadas e tratadas.

Observamos a escassez de estudos abordando essa temática em nível de Ceará, sendo necessário aprofundar conhecimentos, visando a melhoria da prática de enfermagem voltada a tentativas de suicídio, já que mostra-se insuficiente o investimento feito em capacitações e práticas de Educação Permanente para estes profissionais principalmente em unidades de emergência. Além disso, ○ investimento em uma formação direcionada à saúde mental como aspecto transversal ao cuidado pode ser, a longo prazo, uma alternativa para revalorizar e ressignificar a assistência.

Estudos semelhantes são oportunos para a instituição traçando o perfil das pessoas atendidas 
sob esse contexto. Pesquisas paralelas de caráter qualitativo podem ser desenvolvidas o que favorece a promoção de ações que tornem menos prováveis, futuras tentativas.

\section{AGRADECIMENTOS}

Pelo apoio financeiro e institucional à Fundação Cearense de Apoio ao Desenvolvimento Científico e Tecnológico (FUNCAP) e à Santa Casa de Misericórdia de Sobral.

\section{REFERÊNCIAS}

1. Organização Mundial de Saúde (OMS). Relatório mundial da saúde: saúde mental - nova concepção, nova esperança. Genebra. 2001.

2. Krüger LL, Werlang BSG. A dinâmica familiar no contexto da crise suicida. PsicoUSF. 2010;15(1):59-70. doi: 10.1590/S141382712010000100007

3. Macente LB, Zandonade E. Estudo da série histórica de mortalidade por suicídio no Espírito Santo (de 1980 a 2006). J Bras Psiquiatr. 2011 ;60(3):151-7. doi: 10.1590/S004720852011000300001

4. Brasil. Secretaria da Saúde do Estado do Ceará. Doenças e agravos não transmissíveis DANT no Ceará: situação epidemiológica, 1998 a 2004. Fortaleza. Ceará. 2006

5. Macente LB, Santos EG, Zandonade E. Tentativas de suicídio e suicídio em município de cultura Pomerana no interior do estado do Espírito Santo. J Bras Psiquiatr. 2009;58(4):238-244. doi: $10.1590 /$ S0047-20852009000400004

6. Brasil. Ministério da Saúde. Diretrizes Nacionais para Prevenção do Suicídio. Portaria $n^{\circ} 1.876$ de 14 de Agosto de 2006.
7. Brasil. Ministério da Saúde. Secretaria de Atenção à Saúde. Departamento de Ações Programáticas Estratégicas. Manual de Prevenção ao Suicídio dirigido aos profissionais das equipes de saúde mental. Campinas. São Paulo. 2006

8. Organização Mundial da Saúde (OMS). Classificação Estatística Internacional de Doenças e Problemas Relacionados à Saúde - CID-10. Décima revisão. 2008;1.

9. Brasil. Ministério da Saúde. Comissão Nacional de Ética em Pesquisa. Conselho Nacional de Saúde. Normas regulamentadoras de pesquisa envolvendo seres humanos. Resolução $n^{\circ} 466$, de 12 de dezembro de 2012- CNS. Brasília. DF. 2012.

10. Bertolote JM, Mello-Santos C, Botega NJ. Detecção do risco de suicídio nos serviços de emergência psiquiátrica. Rev. Bras. Psiquiatr. 2010;32(suppl.2):S87-S95. doi: 10.1590/S151644462010000600005

11. Alves AAM, Rodrigues NFR. Determinantes sociais e econômicos da Saúde Mental. Rev. Port. Saúde Pública. 2010;28(2):127-131

12. Werlang $B S G$, Borges VR, Fensterseifer $L$. Fatores de risco ou proteção para a presença de ideação suicida na adolescência. Interamerican Journal of Psychology. 2005;39(2):259-266. doi: $10.1590 / 2175-3539 / 2015 / 0193857$

13. Sá NNB, Oliveira MGC, Mascarenhas MDM, Yokota RTC, Silva MMA, Malta DC. Atendimentos de emergência por tentativas de suicídio, Brasil, 2007. Rev. Méd. Minas Gerais. 2010;20(2):14552

14. Abasse MLF, Oliveira RC, Silva T.C, Souza ER. Análise epidemiológica da morbimortalidade por suicídio entre adolescentes em Minas Gerais, Brasil. Ciênc. saúde coletiva. 2009;14(2):407-416. doi: $10.1590 /$ S1 $413-81232009000200010$

15. Santos AS, Lovisi G, Legay L, Abelha L. Prevalência de transtornos mentais nas tentativas de suicídio em um hospital de emergência no Rio de Janeiro, Brasil. Cad. Saúde Pública. 2009;25(9):2064-2074. doi: 10.1590/S0102$311 \times 2009000900020$ 
16. Werneck GL, Hasselmann MH, Phebo LB, Vieira DE, Gomes VLO. Tentativas de suicídio em um hospital geral no Rio de Janeiro, Brasil. Cad. Saúde Pública. 2006;22(10):2201-2206. doi: 10.1590/ S0102-311X2006001000026

17. Sá ACA, Medeiros MFN, Diniz ERS, Silva MLN, Medeiros SS. Percepções dos profissionais de enfermagem acerca do cuidar do paciente suicida. FIEP Bulletin. 201 2;82 